\title{
ESPGHAN Revised Porto Criteria for the Diagnosis of Inflammatory Bowel Disease in Children and Adolescents
}

\author{
${ }^{*}$ Arie Levine, ${ }^{\dagger}$ Sibylle Koletzko, ${ }^{\ddagger}$ Dan Turner, ${ }^{\S}$ Johanna C. Escher, "Salvatore Cucchiara, \\ ${ }^{\S}$ Lissy de Ridder, "Kaija-Leena Kolho, " Gabor Veres, ${ }^{* *}$ Richard K. Russell, \\ ${ }^{\dagger \dagger}$ Anders Paerregaard, ${ }^{\ddagger \dagger}$ Stephan Buderus, ${ }^{\S \S}$ Mary-Louise C. Greer, ${ }^{|||| \uparrow}$ Jorge A. Dias,

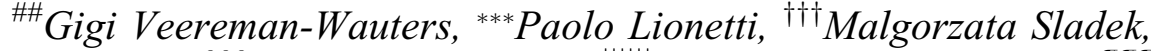 \\ ${ }^{\ddagger \dagger}$ Javier Martin de Carpi, ${ }^{\S \S}$ Annamaria Staiano, ${ }^{\|I\|||}$ Frank M. Ruemmele, and ${ }^{\uparrow \uparrow \uparrow}$ David C. Wilson
}

\begin{abstract}
Background: The diagnosis of pediatric-onset inflammatory bowel disease (PIBD) can be challenging in choosing the most informative diagnostic tests and correctly classifying PIBD into its different subtypes. Recent advances in our understanding of the natural history and phenotype of PIBD, increasing availability of serological and fecal biomarkers, and the emergence of novel endoscopic and imaging technologies taken together have made the previous Porto criteria for the diagnosis of PIBD obsolete.

Methods: We aimed to revise the original Porto criteria using an evidencebased approach and consensus process to yield specific practice recommendations for the diagnosis of PIBD. These revised criteria are based on the Paris classification of PIBD and the original Porto criteria while

incorporating novel data, such as for serum and fecal biomarkers. A consensus of at least $80 \%$ of participants was achieved for all recommendations and the summary algorithm.

Results: The revised criteria depart from existing criteria by defining 2 categories of ulcerative colitis (UC, typical and atypical); atypical phenotypes of UC should be treated as UC. A novel approach based on multiple criteria for diagnosing IBD-unclassified (IBD-U) is proposed. Specifically, these revised criteria recommend upper gastrointestinal endoscopy and ileocolonscopy for all suspected patients with PIBD, with small bowel imaging (unless typical UC after endoscopy and histology) by magnetic resonance enterography or wireless capsule endoscopy.
\end{abstract}

Received October 30, 2013; accepted November 6, 2013.

From the *Pediatric Gastroenterology and Nutrition Unit, Wolfson Medical Center, Sackler School of Medicine, Tel-Aviv University, Tel-Aviv, Israel, the $\dagger$ Dr von Hauner Children’s Hospital, Ludwig Maximilians University, Munich, Germany, the †Pediatric Gastroenterology Unit, Shaare Zedek Medical Center, The Hebrew University of Jerusalem, Israel, the §Pediatric Gastroenterology, Department of Pediatrics, Erasmus MC-Sophia Children's Hospital, Rotterdam, The Netherlands, the ||Pediatric Gastroenterology and Liver Unit, Sapienza University of Rome, Italy, the \Children’s Hospital, University of Helsinki, Helsinki, Finland, the \#Semmelweis University, Budapest, Hungary, the **Department of Paediatric Gastroenterology and Nutrition, Yorkhill Children’s Hospital, Glasgow, UK, the ††Department of Paediatrics, Hvidovre University Hospital, Copenhagen, Denmark, the $\$ \ddagger S t .-M a r i e n-H o s p i t a l$, Department of Pediatrics, Bonn, Germany, the $\S \S$ Department of Diagnostic Imaging, The Hospital for Sick Children, the |||| Department of Medical

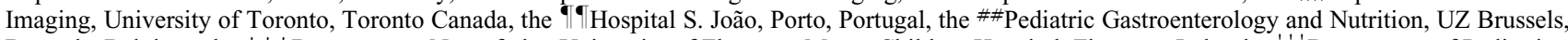
Brussels, Belgium, the $* * *$ Departement Neurofarba, University of Florence, Meyer Children Hospital, Florence, Italy, the $\dagger \dagger \dagger$ Department of Pediatrics, Gastroenterology and Nutrition, Jagiellonian University Medical College, Cracow, Poland, the $¥ \dagger \ddagger$ Department of Pediatric Gastroenterology, Hepatology and Nutrition, Hospital Sant Joan de Déu, Barcelona, Spain, the $\S \S$ Department of Translational Medical Sciences, Section of Pediatrics, University of Naples “'Federico II," Naples, Italy, the |||||||Université Sorbonne Paris Cité, Université Paris Descartes, INSERM U989, AP-HP, Hôpital Necker Enfants Malades, Service de Gastroentérologie Pédiatrique, Paris, France, and the $\uparrow \uparrow \uparrow$ Child Life and Health, University of Edinburgh, Edinburgh, UK.

Address correspondence and reprint requests to Professor David C. Wilson, MD, Child Life and Health, University of Edinburgh, 20 Sylvan Place, Edinburgh EH9 1UW, UK (e-mail: d.c.wilson@ed.ac.uk).

Drs Levine, Koletzko, and Wilson contributed equally to the article.

Drs Levine, Koletzko, Turner, Escher, Cucchiara, de Ridder, and Wilson are steering committee members.

A.L. has received consultation fees, speaker's fees, meeting attendance support or honoraria from MSD, Abbott, Ferring, Falk, and Nestle; S.K. has received consultation fees, speaker's fees, meeting attendance support, or research support from MSD, Abbott, Immundiagnostik, Danone, Janssen, and Nestle; D.T. has received consultation fees, speaker's fees, meeting attendance support, research support, or honoraria from MSD, Abbott, Ferring, Nestle, Shire, and Centocor; J.C.E. has received consultation fees, speaker's fees, meeting attendance support, or research support from MSD and Janssen; S.C. has received consultation fees from MSD and Pfizer; L.d.R. has received consultation fees, speaker's fees, meeting attendance support, or research support from MSD, Abbott, and Shire; K.L.K. has received consultation fees, speaker's fees, or meeting attendance support from MSD, Abbott, Biocodex, and Tillotts Pharma; G.V. has declared no conflicts of interest; R.K.R. has received consultation fees, speaker's fees, meeting attendance support, or research support from MSD, Ferring, Dr Falk, and Nestle; A.P. has received consultation fees and/or speaker from MSD, Nestlé, and BioCare; S.B. has received consultation fees, speaker's fees, or meeting attendance support from Falk-Foundation, MSD, Nestle, Norgine, and Pfrimmer; M.C.G. has reported no conflicts of interest; J.A.D. has reported no conflicts of interest; G.V.-W. has received consultation fees from MSD, Abbott, Mead Johnson, and Novalac; P.L. has received consultation fees, speaker's fees, and meeting attendance support form Abbvie, Danone Reearch, Nestlè, MSD, and Ferring; M.S. has received consultation fees, speaker's fees, meeting attendance support from MSD, Abbott, Ferring, Ewopharma, Nutricia, and Nestle; J.M.d.C. has received consultation fees, speaker's fees, meeting attendance support, or research support from MSD, Abbott, Ferring, Nestle, Otsuka, and Fresenius Kabi; A.S. reports the following-Movetis (advisory board), Valeas (speaker Bureau); D.M.G. Italy (consultant); Mead Johnson (Speaker Bureau); F.M.R. reports the following-advisory board member or speaker for MSD, Jansen and Jansen, Nestlé, Mead Johnson, Danone, and Biocodes; D.C.W. has received consultation fees, speaker's fees, meeting attendance support, or research support from MSD, Abbott, Ferring, Shire, Warner Chilcott, Pfizer, and Nestle.

Copyright (c) 2014 by European Society for Pediatric Gastroenterology, Hepatology, and Nutrition and North American Society for Pediatric Gastroenterology, Hepatology, and Nutrition

DOI: $10.1097 / \mathrm{MPG} .0000000000000239$ 
Conclusions: These revised Porto criteria for the diagnosis of PIBD have been developed to meet present challenges and developments in PIBD and provide up-to-date guidelines for the definition and diagnosis of the IBD spectrum.

Key Words: Crohn disease, diagnosis, inflammatory bowel diseaseunclassified, inflammatory bowel disease, ulcerative colitis

(JPGN 2014;58: 795-806)

\section{INTRODUCTION}

U ntil recently, the diagnosis of inflammatory bowel disease (IBD) in childhood, whose subtypes comprise Crohn disease (CD), ulcerative colitis (UC), and IBD-unclassified (IBD-U, a form of colonic IBD whose features make it impossible to define as either colitis of $\mathrm{CD}$ or $\mathrm{UC}$ at diagnosis), seemed straightforward. The diagnosis of IBD required chronic inflammation in the gastrointestinal (GI) tract and exclusion of other causes of inflammation. The differentiation of CD from UC, and both of these from infectious diseases, allergic diseases, or primary immunodeficiency disorders (PIDs) with similar presentations, was based largely on the clinical suspicion, ruling out other diagnoses, endoscopic and histological evaluation of the mucosa, and small bowel (SB) followthrough (which has limited sensitivity for detecting SB inflammation) (1). Larger and recent data sets of patients with pediatriconset IBD (PIBD) have highlighted several atypical phenotypes of all 3 forms of PIBD, which have led to frequent mislabeling of patients and recognition of the need for more accurate definitions of each subset of disease (2-8). The Paris classification (8) was a significant step forward in the standardization of definitions and classification of PIBD. Advances in diagnostic imaging modalities, capsule endoscopy, and comprehensive serological and fecal biomarkers, have also boosted our ability to detect and characterize these diseases while reducing radiation exposure in children, but have themselves presented new challenges. These modalities increased not only the sensitivity of mucosal lesion detection but also the uncertainty in some children with the isolated colitis phenotype who have perceived overlapping features. Thus, the accurate diagnosis of PIBD depends not only on an index of suspicion and choice of tests, but also on the appropriate interpretation of the results of the workup. This present revision of the original Porto Criteria of 2005 (1) uses an evidence-based approach to meet our goals - not only to facilitate the diagnosis of PIBD but also to enable clinicians to properly diagnose each individual subtype based on evidence. The new criteria integrate the most recent evidence regarding the recommended methods for diagnosis of IBD, clearly define the disease subtypes of PIBD based on the Paris phenotypic classification, and highlight the diagnostic pitfalls to provide reliable diagnosis, assessment, and prognosis, leading to the best individualized care for a new generation of patients with PIBD. Using a novel, evidence-based approach, we have confronted, in particular, the difficult issue of defining and evaluating IBD-U. Although esophagogastroduodenoscopy (EGD) and ileocolonoscopy are tests that are within the broad consensus for the initial evaluation of PIBD irrespective of disease type, the choice of additional tests depends on phenotypes and interpretation of endoscopic data. Thus, these revised Porto criteria start with a description of phenotypes and pitfalls in interpretation of the data to guide a physician or surgeon in the choice of investigations.

\section{METHODS}

An international group of European experts in PIBD, mainly from the "Porto" IBD Working Group of the European Society of
Pediatric Gastroenterology, Hepatology, and Nutrition (ESPGHAN), wished to construct a revised, methodologically robust, consensus-based clinical guideline for the diagnosis of PIBD, including full facets of the assessment by all investigative modalities, and interpretation of these results. This was to build on and update the earlier work, which is now known as the 'Porto criteria' (1), and aimed to comprise the best recent available evidence from the PIBD literature, relevant methodologically high-quality data from the adult IBD literature, together with clinical expertise from PIBD specialists, based on the work in their multidisciplinary IBD teams. A major reference point was the Paris classification (8), an expert-consensus document providing a pediatric-specific modification of the Montreal classification of IBD (9), which specifically has highlighted those phenotypic characteristics that are either more common in or unique to pediatric-onset rather than adult-onset IBD.

A list of 12 topics addressing the diagnosis of PIBD (age at diagnosis $<17$ years) was developed by the steering committee and modified according to the comments by other members of the working group. Each topic was assigned to a subgroup of 2 to 3 members to draft an initial document based on a complete literature review. Electronic searches were performed in summerautumn 2011 using MEDLINE, PubMed, Embase, CINAHL, and the Cochrane Controlled Trials Register, along with perusal of reference lists from the literature and participants' personal collections. Clinical guidelines, systematic reviews, clinical trials, cohort studies, case-control studies, diagnostic studies, surveys, letters, narrative reviews, and case series were retrieved and appraised. Grading of evidence and recommendations followed the pattern of all recent ESPGHAN PIBD and European Crohn's and Colitis Organisation IBD guidelines and were assigned according to the Oxford Centre for Evidence-Based Medicine (http://www.cebm.net/ levels_of_evidence.asp\#refs).

The group met twice in Stockholm, Sweden, in October 2011 (during United European Gastroenterology Week), and in April 2012 (during ESPGHAN). In the initial face-to-face meeting, each of the above topics were discussed fully, and areas of consensus and need for reconsideration emerged. The meetings were complemented by an e-mail-consensus process until an agreement was reached on the recommendations and the draft proposals, which had been reviewed 3 times by all of the authors. By the end of this iterative process, electronic voting on the recommendations and the summary algorithm (Fig. 1) achieved $>80 \%$ consensus. Key new evidence published in 2012 had been considered for inclusion in relevant sections in autumn 2012. The final manuscript has been approved by all of the participants. The guidelines include not only recommendations but also "practice points" that reflect common practice wherein evidence is lacking.

\section{PART I: DIAGNOSIS OF IBD}

\section{Recommendations}

Accurate diagnosis of inflammatory bowel disease (IBD) should be based on a combination of history, physical and laboratory examination, esophagogastroduodenoscopy (EGD) and ileocolonoscopy with histology, and imaging of the small bowel. It is critical to exclude enteric infections. [EL2b, RGC]

We recommend performing small bowel imaging in all suspected cases of IBD at diagnosis; this may be deferred in typical $U C$, based on endoscopy and histology. Imaging is particularly important in suspected Crohn's disease, in patients whose ileum could not be intubated, in patients with apparent ulcerative colitis with atypical presentations, and in patients with IBD-unclassified. [EL3, RGD] 


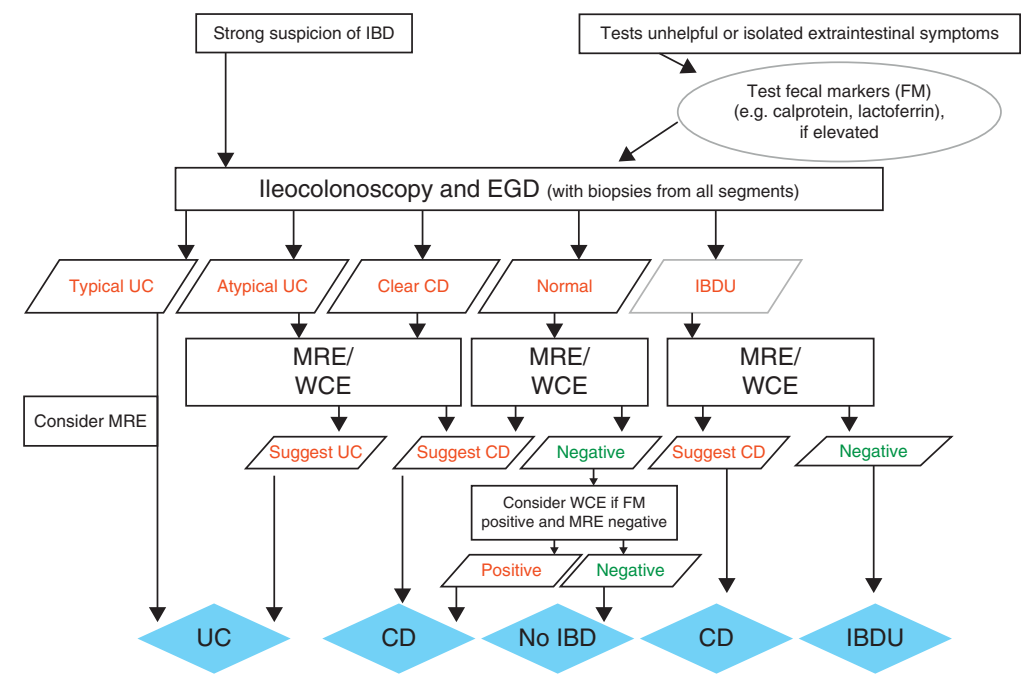

FIGURE 1. Evaluation of child/adolescent with intestinal or extraintestinal symptoms suggestive of IBD. Atypical UC is a new IBD category consisting of 5 phenotypes defined in Table 1, and reflects a phenotype that should be treated as UC. IBD-U may be entertained as a tentative diagnosis after endoscopy, and can be used as a final diagnosis after imaging and a full endoscopic workup. UC is divided into typical UC and atypical UC. $C D=$ Crohn disease; $E G D=$ esophagogastroduodenoscopy; $F M=$ fecal marker; IBD = inflammatory bowel disease; $M R E=$ magnetic resonance enterography; $\mathrm{UC}=$ ulcerative colitis; $\mathrm{WCE}=$ wireless capsule endoscopy.

\section{Practice Points}

1. The most important reliable feature of typical UC is continuous mucosal inflammation of the colon, starting distally from the rectum, without SB involvement other than backwash ileitis, and without epithelioid granulomas on biopsy. Disturbed crypt architecture and focal or diffuse basal plasmacytosis are characteristic.

2. Pediatric-onset UC may present with atypical phenotypes such as macroscopic rectal sparing, isolated nonserpiginous gastric ulcers, normal crypt architecture, absence of chronicity in biopsies, or a cecal patch. Patients with acute severe colitis may have transmural inflammation. These individual phenotypes in isolation should not lead to reclassification to $C D$.

3. Patients with disease limited to the colon with class 1 findings (Table 3) should be classified as CD.

4. IBD-U should be the preferential classification for patients with colitis and highly atypical findings (defined as class 2 or class 3 findings inTable 3 ) for either $\mathrm{CD}$ or UC, or a combination of findings presented below.

IBD should be suspected when patients appear with the appropriate symptoms, which may be extremely diverse (10-13). Bloody diarrhea is the most common presenting symptom in UC whereas $C D$ may present with vague abdominal pain, diarrhea, unexplained anemia, fever, weight loss, or growth retardation as frequently reported symptoms. The classic "triad"' of abdominal pain, diarrhea, and weight loss occurs in only $25 \%$ of patients with $\mathrm{CD}$ (14). Extraintestinal manifestations may present at diagnosis in $6 \%$ to $23 \%$ of children with a higher frequency in those $>6$ years $(3,12,15)$. It is beyond the scope of this article to report the complete list of luminal or extraintestinal symptoms or presentations of IBD.

The classification of IBD is complex and characterized by many rare phenotypes that are atypical or unusual. It requires the recognition of the typical features of $\mathrm{CD}$ and $\mathrm{UC}$, identification of atypical phenotypes that are still consistent with a diagnosis of CD or UC, and knowledge of those factors that preclude a diagnosis of one or the other.

\section{Diagnosis and Classification of UC}

The diagnosis of UC relies on the identification of a typical phenotype of chronic inflammation of the colon upon colonoscopy and colonic biopsies, and the exclusion of both $\mathrm{CD}$ and infectious causes of colitis. On the contrary, there is no single set of macroscopic or microscopic criteria that can accurately diagnose UC, and there are multiple atypical phenotypes that do not fit into this category. Features of typical and 5 atypical variants of pediatric UC appear in Table 1. This discussion will therefore focus initially on the typical phenotype and then delineate the features of atypical UC in children.

The most reliable feature to diagnose UC is continuous mucosal inflammation of the colon, starting from the rectum, without SB involvement, and without granulomas on biopsy $(2,6,16)$. Typical macroscopic features of UC include erythema, granularity, friability, purulent exudates, and ulcers that usually appear as superficial small ulcers (16). The inflammation may either end at a transition zone anywhere in the colon or involve the whole colon continuously. The most distal part of the terminal ileum may show nonerosive erythema or edema if pancolitis is present and the ileocecal valve is involved (termed "backwash ileitis"), but should be normal in all other circumstances. Disturbed crypt architecture and focal or diffuse basal plasmacytosis are signs of chronicity and thus are good predictors of IBD occurring in $70 \%$ of adult patients with IBD and $<5 \%$ of patients with infectious colitis (17). The chronic inflammation is often accompanied by cryptitis or crypt abscesses. Typically, inflammation is most severe distally and a reverse gradient (ie, severe proximal inflammation and mild distal inflammation) should prompt the reconsideration of the diagnosis of $\mathrm{UC}$, except in cases of macroscopic rectal sparing that is occasionally seen in UC $(5,18)$.

The historical perception that considered UC as a superficial inflammatory disease confined to the colon, uniformly involving the rectum and progressing contiguously to varying degrees, has been found to be simplistic in the pediatric age group, and 5 atypical variants should be recognized:

1. The best defined atypical presentation is macroscopic rectal sparing in untreated patients, which has been reported in $5 \%$ to 


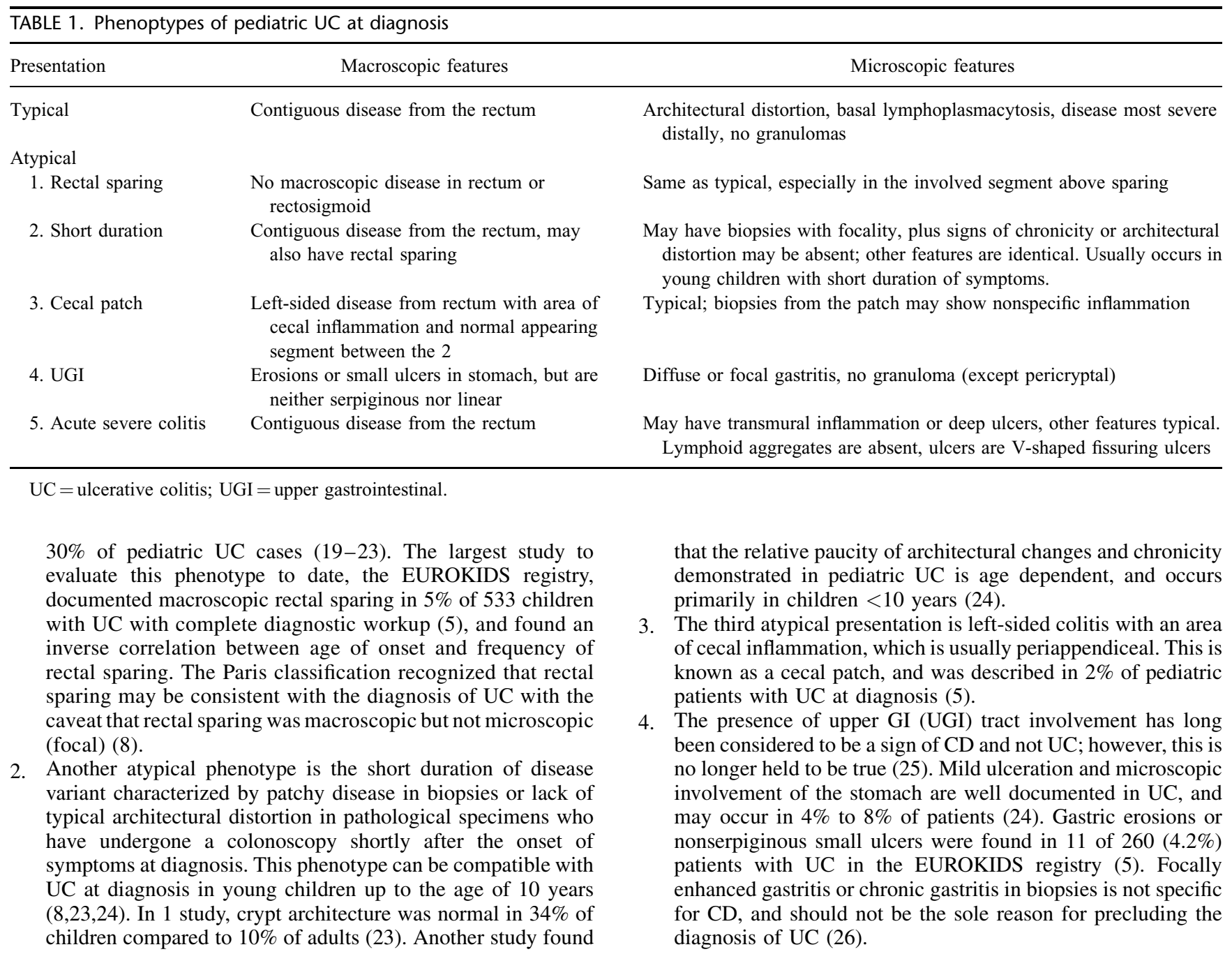

TABLE 2. Macroscopic and microscopic features of untreated pediatric luminal CD

\section{Typical macroscopic findings of $\mathrm{CD}$ : \\ Mucosal aphthous ulcers \\ Linear or serpentine ulceration}

\section{Cobblestoning}

Stenosis/stricturing of bowel with prestenotic dilatation

Imaging or surgical—bowel wall thickening with luminal narrowing

Perianal lesions-fistula(s), abscesses, anal stenoses, anal canal ulcers, large and inflamed skin tags

Skip lesions

Jejunal or ileal ulcers

Nonspecific macroscopic findings of CD

Oedema

Erythema

Friability

Granularity

Exudate

Loss of vascular pattern

Isolated aphthous ulcers

Perianal lesions-midline anal fissures, small skin tags
Typical microscopic findings of $\mathrm{CD}$ :

Noncaseating granuloma(s) - must be remote from ruptured crypt

Focal chronic inflammation, transmural inflammatory infiltrate, submucosal fibrosis

$\mathrm{CD}=$ Crohn disease 
TABLE 3. Diagnostic features in a child with untreated colitis phenotype at diagnosis

Likelihood of

occurring in UC

Feature

Diagnostic approach

Class 1: Nonexistent

Class 2: Rare with UC $(<5 \%)$

Class 3: Uncommon $(\sim 5 \%-10 \%)$
Well-formed granulomas anywhere in the GI tract, remote from ruptured crypt

Deep serpentine ulcerations, cobblestoning or stenosis anywhere in the SB or UGI tract

Fistulizing disease (internal or perianal)

Any ileal inflammation in the presence of normal cecum (ie, incompatible with backwash ileitis)

Thickened jejunal or ileal bowel loops or other evidence of significant SB inflammation (more than a few scattered erosions) not compatible with backwash ileitis*

Macroscopically and microscopically normal appearing skip lesions in untreated IBD (except with macroscopic rectal sparing and cecal patch)

Large inflamed perianal skin tags

Combined (macroscopic and microscopic) rectal sparing, all other features are consistent with UC

Significant growth delay (height velocity $<2$ SDS), not explained by other causes

Transmural inflammation in the absence of severe colitis, all other features are consistent with UC

Duodenal or esophageal ulcers, not explained by other causes (eg, Helicobacter pylori, NSAIDs and celiac disease)

Multiple aphthous ulcerations in the stomach, not explained by other causes (eg, $H$ pylori and NSAIDs)

Positive ASCA in the presence of negative pANCA

Reverse gradient of mucosal inflammation (proximal > distal (except rectal sparing))

Severe scalloping of the stomach or duodenum, not explained by other causes (eg, celiac disease and $H$ pylori)

Focal chronic duodenitis on multiple biopsies or marked scalloping of the duodenum, not explained by other causes (eg, celiac disease and $H$ pylori)

Focal active colitis on histology in more than 1 biopsy from macroscopically inflamed site

Non-bloody diarrhea

Aphthous ulcerations in the colon or UGI tract
Diagnose as $\mathrm{CD}$

Diagnose as IBD-U, if at least 1 class 2 feature exists**

Diagnose as IBD-U if at least $2-3$ features exist

ASCA = anti-Saccharomyces cerevisiae antibody; $\mathrm{GI}=$ gastrointestinal; $\mathrm{IBD}=$ inflammatory bowel disease; $\mathrm{NSAID}=$ nonsteroidal anti-inflammatoy drug; pANCA = perinuclear antineutrophil cytoplasmic antibody; $\mathrm{SB}=$ small bowel; $\mathrm{SDS}=$ standard deviation score; $\mathrm{UC}=$ ulcerative colitis .

* After full diagnostic workup including SB imaging.

** The likelihood of CD increases with increasing number of class 2 features.

5. Children presenting with acute severe UC at presentation may have several features that are typically characteristic of $\mathrm{CD}$, such as transmural inflammation and deep ulcers, reflecting the severity of the disease and not the type of disease. Certain histopathological features such as the shape of the ulcers (V shaped, with fissuring) or absence of lymphoid aggregates are supportive of severe UC, as opposed to CD (16). Ultimately, these patients will be diagnosed by the combination of clinical, pathological, and endoscopic and serological features, and may be diagnosed in uncertain cases as IBD-U until the disease develops its characteristic features over time (Table 3 ).

\section{Terminal Ileal Involvement in UC}

The short segment of nonstenosing mild macroscopic terminal ileitis without granulomata (termed backwash ileitis) occurs in $6 \%$ to $20 \%$ of patients with UC with pancolitis, and this has been demonstrated also in children (27-30). The most common histological feature of backwash ileitis consists of patchy areas of neutrophilic cryptitis without surface ulceration, but superficial small ulcers, a mild degree of villous atrophy, and lymphocytic infiltration in the lamina propria may be seen in one-third of cases $(29,30)$.

\section{Diagnosis of CD}

Typical features of pediatric CD have been fully described in the Paris classification (8) (Table 2). These include noncontiguous aphthous or linear ulcers primarily in the ileum or colon, although $\mathrm{CD}$ may involve any area of the GI tract, and disease may be confluent. CD may present with extraintestinal manifestations initially; in this scenario the definitive diagnosis requires evidence of GI disease. Histologically the disease is usually characterized by chronic focal inflammation, with or without granulomas (Table 2); recognition of granulomatous inflammation is vital in the diagnosis of CD (31).

The diagnosis may become more difficult in cases of infantile-onset IBD (0-2 years) (8) and in cases with predominantly colonic disease, in which confusion may arise with UC or IBD-U $(6,8)$. Rare phenotypic presentations of $\mathrm{CD}$ can be arbitrarily defined as those occurring in $<5 \%$ of cases. In a detailed report of the differences in phenotype at diagnosis and follow-up of 416 Scottish children and 1296 adults with IBD, Van Limbergen et al (2) reported that $5 \%(14 / 273)$ of CD children at diagnosis had oral and perianal, isolated perianal, or isolated oral $\mathrm{CD}$ alone without any evidence for GI luminal disease, of whom $70 \%$ developed luminal disease at $\geq 4$ years follow-up. Conventionally, the term orofacial granulomatosis (OFG) is used to describe patients with granulomatous oral lesions, but without the evidence of $\mathrm{CD}$ elsewhere in the lumen of the GI tract. In contrast, patients with intestinal CD who have involvement of the mouth typically are described as having oral CD (29). OFG in childhood, most often reported in Celtic populations $(2,32)$, is usually associated with rapid development of luminal $\mathrm{CD}$, and oral features are frequently lost as follow-up progresses (32); adult presentation of OFG is less likely 
to predict the development of CD (33). Isolated perianal disease with granulomas on biopsy can also present as either silent or rapidly developing luminal CD. In a large North American series, $10 \%$ of newly diagnosed pediatric patients with CD had perianal fistulas and/or abscesses at diagnosis (34). Genital lymphoedema with granulomas at skin biopsy is recognized as a metastatic form of $\mathrm{CD}$ (35), and may be the presenting symptom in up to $1.5 \%$ of children with CD (D.C. Wilson, unpublished observation). Extraintestinal manifestations were present in $20 \%$ of 1178 cases of incident CD in EUROKIDS, a large prospective European (17 European countries and Israel) PIBD registry (36).

In terms of differentiation of colonic CD from IBD-U or UC, key features that point only to the diagnosis of $\mathrm{CD}$ appear in Tables 2 and 3. These key features include the presence of skip lesions; the presence of well-formed noncaseating granulomas remote from ruptured crypts anywhere in the gut; the presence of macroscopic lesions of the upper intestinal tract, in particular serpentine ulcers and cobblestoning (1); stenosis/stricturing of bowel (radiological or surgical)_bowel wall thickening with luminal narrowing; stenosis or cobblestoning, and linear ulcerations in the ileum, or inflamed ileum with a normal cecum.

The presence of epithelioid granulomas on biopsy from an area of the GI tract with nondiagnostic macroscopic CD-like appearance (see Table 2) is sufficient to define the diagnosis of $\mathrm{CD}$ in a case that otherwise would be labeled as UC or IBD-U, that is, once granulomas are found, both microscopic and macroscopic changes can define CD locations (2). Granulomas are more common in childhood-onset rather than adult-onset Crohn colitis at diagnosis, and tend to regress, frequently being absent in surgical specimens if surgery is later required (37). The presence of granulomas saves confusion in rare presentations, such as when CD presents with duodenal villous atrophy, or their absence can be helpful in the reverse situation, when another GI disease process has clinical features suggestive of $\mathrm{CD}$, such as the occurrence of duodenal ulcerations in celiac disease.

\section{Diagnosis of IBD-U}

Typically, IBD type unclassified (IBD-U) is a term referring to patients with definite IBD, wherein the inflammation is limited to the colon with features that make the differentiation between UC and CD uncertain even after a complete workup (38). Some phenotypic findings may be described with either CD or UC, but because the follow-up period is limited, misclassification bias may exist. For instance, in a study of pediatric patients undergoing ileal pouch-anal anastomosis, 17 of 125 (14\%) patients with a perioperative diagnosis of UC were rediagnosed as having CD after a follow-up period of 5 years (39).

Table 3 suggests a general scheme for the use of features that are consistent with CD or UC, atypical phenotypes or tests that are still consistent with a diagnosis of UC or CD (along with Tables 13 ), and variables that should trigger the diagnosis of IBD-U. There is no absolute rule as to how many adjuvant variables should trigger the diagnosis of IBD-U. Extra care should be made in diagnosing $\mathrm{UC}$ in the isolated colitis phenotype $<5$ years, and the threshold for labeling an infant with colitis as IBD-U should be lower than in adolescents. Height velocity $<2$ age-related standard deviations of the norm is rare in UC. Among 205 consecutive children with UC (2-18 years), mean $z$ score for height at diagnosis was $-0.1 \pm 1.1$ (ie, normal Gaussian distribution) with only 8 children having $z$ score $<2$ SDS (4\%), just above that expected from a normal curve (D. Turner and A.M. Griffiths unpublished data). These findings are consistent with other published pediatric data (8). Although atypical, the absence of rectal bleeding does not preclude the diagnosis of UC because $5 \%$ to $-15 \%$ of adults and children with UC may present with non-bloody diarrhea $(8,40,41)$. The presence of non-bloody diarrhea at diagnosis of adults with UC, however, was a strong predictor for eventually changing the classification to $\mathrm{CD}(40 \%$ nonbloody diarrhea in those who changed classification versus $4 \%$ in others, $P<0.001$ ) (40).

\section{Endoscopic and Histological Features}

Aphthous ulcerations are typical of $\mathrm{CD}$ and are rarely seen also in UC; however, deep serpentine ulcers and cobblestoning anywhere in the GI tract characterize CD (42). Skip lesions are not typical of UC, but focality and absence of chronicity in biopsies are not uncommon especially in young children at diagnosis or during treatment $(21,22)$. Histologically, normal mucosa between inflamed segments, however, precludes the diagnosis of $\mathrm{UC}-$ with the exception of left-sided colitis with a cecal patch (18-21).

Macroscopic rectal sparing may coexist with UC as noted above. Some of these patients with the absence of histological inflammation (microscopic sparing) proved to have $\mathrm{CD}$, years after the initial diagnosis (19), and microscopic rectal sparing was a predictor for eventually changing the diagnosis to CD (43). The severity of backwash ileitis in UC correlates with the degree of inflammation in the right colon $(28,29)$; thus, the presence of severe ileal inflammation with mild colitis argues against the diagnosis of UC (28). Similarly, ileitis in the presence of normally looking cecum, or the presence of ileal fissuring ulcers, is consistent with the diagnosis of CD (30). A few small erosions in the UGI tract or in the SB (found on capsule endoscopy) do not preclude the diagnosis of UC because these may be found in a significant proportion of healthy individuals, and also because some degree of nonspecific UGI and SB inflammation is allowed in UC.

The presence of focal active colitis in multiple biopsies of inflamed areas (ie, isolated finding of focal infiltration of the colonic mucosa by neutrophils) is not consistent with the established untreated UC. In a study of 29 children with this finding at diagnosis, $8(28 \%)$ eventually developed CD and only $1(3 \%)$ developed UC (44). Isolated biopsies with focal colitis, however, have been noted in patients with new-onset UC of short duration (18), with no subsequent change in diagnosis of UC over time.

\section{Serology and Subtype of IBD}

No serology pattern can preclude the diagnosis of UC because of imperfect test performance of all existing antibodies. The presence of positive Crohn serology (eg, anti-Saccharomyces cerevisiae antibody [ASCA] $+/ \mathrm{pANCA}-$ ) does not necessarily preclude the diagnosis of UC but reduces the likelihood of UC. ASCA is found more often in CD $(50 \%-70 \%)$ than in UC $(10 \%-$ $15 \%)$ and healthy controls $(<5 \%)(45,46)$; these antibodies increase with age $(47)$ and are associated with a more severe disease course in $\mathrm{CD}(48,49)$. ASCA positivity is not typically present in isolated colitis. pANCA is more common in UC $(60 \%-70 \%)$ than in $\mathrm{CD}$ $(20 \%-25 \%)(50)$. The presence of pANCA + /ASCA - serology in patients with isolated colitis is not helpful for diagnosing specific phenotypes; among 20 patients who had pANCA+/ASCA- results at baseline, 4 were later diagnosed with $C D$ and 7 with UC (50). In a prospective follow-up of IBD-U patients, Joossens et al (51) found 26 patients who were ASCA+/pANCA - at baseline; 8 were later diagnosed with $C D$ and 2 with UC. The reverse profile was even less helpful for definitive diagnosis. In IBD-U, a significant number of patients seem to have negative serology, but this provides no help in the diagnostic process (49).

Newer serological markers (which include antibodies against Pseudomonas fluorescens-associated sequence [anti-I2], antiouter membrane protein $\mathrm{C}$ of Escherichia coli [anti-OmpC], antiouter 
membrane protein of Bacteroides caccae [anti-OmpW], and antiflagellin antibodies [anti-CBir1]) may be detected in children who otherwise have negative serology (47,52-54); all these markers are nonspecific and can be detected in patients with other diseases.

\section{PART 2: DIAGNOSTIC WORKUP OF CHILDREN WITH SUSPECTED IBD}

This section deals with diagnostic methods recommended in children with suspected IBD. Recommendations and practice points are designed to assist with confirmation of the diagnosis, assessment of disease location, extent, and severity, as well as for recognizing complications at diagnosis. It is beyond the scope of these guidelines to recommend tests needed for the management or follow-up.

\section{Recommendation}

In children with suspected IBD, enteric infections should be excluded as cause of the symptoms preferentially before endoscopy is performed. Microbiological investigation should exclude bacterial infections including Clostridium difficile. [EL2, RGC]

\section{Practice Points}

1. A more extensive investigation for unusual infective agents and parasites may be warranted in endemic areas or after travel.

2. The search for bacterial infections should include a stool culture to exclude Salmonella, Shigella, Yersinia, Campylobacter, and Clostridum difficile toxins in all of the children. Screening for enteric viruses is rarely helpful. Testing for Giardia lamblia is recommended in high-risk populations or endemic areas.

3. The identification of a pathogen does not necessarily exclude a diagnosis of IBD because a first episode or flare of IBD may be triggered by a documented enteric infection.

\section{Recommendation}

Initial blood tests should include complete blood count, at least two inflammatory markers, albumin, transaminases and $\gamma G T$. Fecal calprotectin is superior to any blood marker for detection of intestinal inflammation (EL2, RGC).

\section{Practice Points}

1. Normal blood tests do not exclude the diagnosis of IBD.

2. A low serum albumin may indicate protein losing enteropathy, and usually reflects disease activity as well as severity, and not merely nutritional status.

3. Fecal markers (FMs) of inflammation (eg, calprotectin or lactoferrin) are extremely sensitive in the detection of mucosal inflammation, but are not specific for IBD; fecal calprotectin $(F C)$ is a more sensitive tool for diagnosis in new-onset IBD patients than serum inflammatory markers such as $C$-reactive protein $(C R P)$ and erythrocyte sedimentation rate (ESR), and normal FC values make active disease in the small or large bowel less likely.

4. Although results are often negative, screening for serological markers of IBD (eg, ASCA, pANCA) may increase the likelihood for IBD in atypical cases if results are positive and may help at times to differentiate $C D$ from $U C$ in cases of IBD- $U$.

5. Additional testing may be required when extraintestinal manifestations such as pancreatitis, uveitis, arthritis or sclerosing cholangitis are present or suspected.
Multiple laboratory tests may be abnormal in IBD and include stool tests to exclude enteric infections, and blood tests-complete blood cell count (decreased hemoglobin or elevated total white cell count and platelet count), serum albumin (decreased), and inflammatory markers such as CRP and ESR, both of which are typically elevated in active disease. Data from pediatric IBD registries indicate that at the time of diagnosis $54 \%$ of children with mild UC and $21 \%$ of children with mild CD have normal results for the combination of hemoglobin, albumin, CRP, and ESR diagnosis (55).

Fecal surrogate markers for detection of inflammation at diagnosis include FC, lactoferrin, S 100 A12, and lysozyme. Pediatric data exist primarily for FC and lactoferrin. Both markers are excellent tools for identifying the presence of intestinal inflammation with high sensitivity $(56,57)$. In a recent prospective study FC was elevated at diagnosis in $95 \%$ of 60 unselected pediatric patients with CD whereas only $86 \%$ of the patients had an increased CRP and $83 \%$ an elevated ESR (58). The combination of any 2 of these 3 markers had higher sensitivity (58-61); in 1 series, $15 \%$ of 48 incident IBD cases had no elevation of any of 5 blood markers (hemoglobin, white cell count, platelet count, ESR, and CRP) yet had abnormally raised FC (62). FC levels at diagnosis of pediatric IBD are superior to blood markers as a diagnostic marker for intestinal inflammation, and discriminate IBD from other extraintestinal inflammatory conditions as well as intestinal noninflammatory conditions. In a recent large case-control study of $\mathrm{FC}$, area under the receiver operating characteristic curve of FC to diagnose IBD was 0.93, considerably higher than the AUC of blood inflammatory markers (63). However, elevated FC levels cannot distinguish between the different causes of intestinal inflammation (eg, IBD vs infection), the type of IBD (CD vs UC), or location of the disease (small vs large bowel), and may occur in apparently healthy infants and toddlers (64). The most recent systematic review and meta-analysis of FC for suspected pediatric IBD contains 394 pediatric IBD patients and 321 non-IBD controls and demonstrated pooled sensitivity and specificity for the diagnostic utility of FC during these investigations of 0.978 ( $95 \%$ confidence interval $0.947-0.996)$ and 0.682 (95\% confidence interval $0.502-0.863$ ), respectively (65). Some patients with enteropathies such as celiac disease or allergic enteropathy may have mildly elevated FC. In the diagnostic algorithm (Fig. 1), FMs may be particularly helpful in children with nonspecific symptoms (eg, abdominal pain or nonbloody diarrhea) or signs (eg, anemia, elevated CRP or ESR) to favor or avoid endoscopic workup. They may also be useful in patients with extraintestinal manifestations without GI symptoms in whom elevated CRP or ESR is unhelpful for discriminating between a primary extraintestinal disorder (eg, arthritis, erythema nodosum in rheumatological diseases) and the same IBD-associated symptoms. In this scenario, elevated FMs should prompt a GI workup. Other fecal tests (eg, for occult blood or fecal $\alpha$-1-antitrypsin) are not recommended for the routine initial diagnostic workup.

Examination of transaminases and $\gamma \mathrm{GT}$ and an ophthalmic examination should be performed to screen for IBD-associated extraintestinal disease such as uveitis and hepatobiliary disease such as primary sclerosing cholangitis (66); abnormal test results may, however, be elevated due to other causes. Other laboratory tests such as anti-tissue transglutaminase immunoglobulin A to exclude celiac disease (67) or tests for immunodeficiency disorders are reserved for use in appropriate circumstances and therefore are not recommended as routine in all of the patients with suspected IBD.

\section{Recommendations}

Ileocolonoscopy and esophagogastroduodenoscopy (EGD) are recommended as the initial work up for all children with suspected IBD. [EL4, RGD] 
Multiple biopsies (2 or more per section) should be obtained from all sections of the visualized gastrointestinal tract, even in the absence of macroscopic lesions. Endoscopic findings should be well documented. [EL5 RGD]

\section{Practice Points}

1. Endoscopy should be performed by a pediatric gastroenterologist after an age-appropriate preparation, under general anesthesia or deep sedation in a setting suited for children, and by personnel with training and expertise in pediatric IBD.

2. The diagnostic yield of an upper EGD with multiple biopsies to diagnose $C D$ in patients with an otherwise normal workup (ileocolonoscopy and SB imaging) is $\sim 7.5 \%$ (number needed to treat $=13$ ).

The recommendations for the initial evaluation of IBD are presented in Figure 1. In nonemergency situations, the workup should start with an upper and lower GI endoscopy. Ileocolonoscopy (and biopsies) is the most essential part of the diagnostic workup in pediatric IBD. Rectosigmoidoscopy and incomplete colonoscopy are insufficient. Failure to visualize the terminal ileum has been reported in $\sim 10 \%$ in experienced large pediatric centers. The diagnostic yield of ileocolonoscopy including histology is reported to be $16.7 \%$ to $19 \%$ in adult patients (17) and $13 \%$ in PIBD (68).

Regarding the number of biopsies, European Crohn's and Colitis Organisation consensus statements on diagnosis and management of both CD (69) and UC (16) recommend that "multiple", biopsies from 5 sites around the colon (including the rectum) and the ileum should be obtained for a reliable diagnosis. A minimum of 2 samples from each of these 6 sites should be obtained.

The previous Porto criteria have advocated EGD (with 2 or more biopsies from the esophagus, stomach and duodenum) to be performed in all children irrespective of presence or absence of UGI symptoms (1). In an audit of diagnostic workup in 1811 pediatric patients with IBD, $35 \%$ of the patients with $\mathrm{CD}$ had macroscopic abnormalities at EGD, and these abnormalities were specific for CD (aphthae, ulcerations, cobblestoning, and stenosis) in $24 \%$ of the patients (68). Microscopic abnormalities on EGD were crucial for the diagnosis of $\mathrm{CD}$ in 19 of 428 patients (4.5\%), including the isolated detection of granuloma at EGD in 13 of 428 patients $(3 \%)$. In a recent review, the isolated detection of granuloma at EGD in pediatric patients with $\mathrm{CD}$ ranged from $2 \%$ to $21 \%$ (70). UGI endoscopy was particularly helpful in patients with otherwise nonspecific pancolitis (71).

\section{Recommendations}

Magnetic resonance enterography (MRE) is currently the imaging modality of choice in pediatric IBD at diagnosis. It may detect small intestinal involvement, inflammatory changes in the intestinal wall and identify disease complications (fistula, abscess, stenosis). MRE is preferred over CT and fluoroscopy because of high diagnostic accuracy and the lack of radiation involved. [EL2, RGC]

Wireless capsule endoscopy (WCE) is a useful alternative to identify small bowel mucosal lesions in children with suspected Crohn disease, in whom conventional endoscopy and imaging tools have been nondiagnostic (EL3b; RGC) or in whom MRE can not be performed due to young age or in settings where MRI is not available or not feasible. A normal WCE study has a high negative predictive value for active small bowel CD. [EL4, RGD]

Ultrasonography is a valuable screening tool in the preliminary diagnostic workup of pediatric patients with suspected IBD, but should be complemented by more sensitive imaging of the small bowel. [EL3, RGC]

\section{Practice Points}

1. Magnetic resonance enterography (MRE) can estimate both the intestinal inflammation and the degree of damage, but there is no validated scoring system for this modality in pediatric patients. SB wall thickening is sensitive, but neither pathognomonic nor specific for $C D$.

2. Although SB imaging is encouraged in all of the patients with suspected IBD, it is essential in pediatric patients with $C D$, $I B D-U$, or atypical UC. In children with a clear macroscopic and histological diagnosis of UC based on ileocolonoscopy and EGD with multiple biopsies, SB imaging can be omitted at diagnosis.

3. During ultrasonography(US), the use of oral anechoic contrast solution (iso-osmolar polyethylene glycol) in the so-called small intestine contrast US enhances the sensitivity and decreases the interobserver variability.

4. Magnetic resonance (MR) colonography has, as yet, no role in the diagnostic workup of PIBD.

5. Imaging tools or a patency capsule should generally precede wireless capsule endoscopy (WCE) to reduce the risk of retention. The choice depends on local availability and expertise.

6. Caution should be borne when WCE is the only SB imaging, due to the high number of false-positives and a lack of validated diagnostic criteria. False-positive features are found in $10-21 \%$ of healthy persons, particularly with nonsteroidal anti-inflammatoy drugs use.

7. Balloon-assisted enteroscopy (BAE) is indicated in special circumstances only (eg, if conventional endoscopy and WCE as well as cross-sectional imaging modalities do not allow a definite $C D$ diagnosis in patients with suspected disease of the $S B$ ).

\section{US}

Noninvasiveness, low-cost, and widespread availability make abdominal US a useful modality for IBD imaging, especially for screening for CD. Several studies have shown that US accurately detects, locates, and characterizes inflammation of the bowel wall and assesses peri-intestinal abnormalities, with a good negative predictive value for IBD, higher for CD than for UC. The pathological changes of inflamed bowel can be essentially divided into mural and extramural findings (72). The latter involve the surrounding mesentery that appears thickened and hyperechoic with adipose tissue alteration and enlarged mesenteric lymph nodes (73). Mural changes occur in the bowel wall, which may be thickened and may show altered echogenicity (hypo- or hyperechogenicity), loss of stratification, increased color-Doppler signal denoting hyperemia, and relative decrease or lack of peristalsis as a marker of stiffness. Different wall thickness values are suggested as threshold for a positive diagnosis in various reports (from 1.5 to $3 \mathrm{~mm}$ for the terminal ileum and $<2 \mathrm{~mm}$ for the colon) (72). Comparative studies between bowel US and ileocolonoscopy and histology in detecting $\mathrm{CD}$ lesions at the terminal ileum have shown an overall sensitivity and specificity of $74 \%$ to $88 \%$ and $78 \%$ to $93 \%$, respectively (74). US is sensitive for detecting lesions of the terminal ileum with decreased sensitivity for proximal SB lesions and colonic lesions. Interobserver variability remains a major issue; small intestine contrast US may increase the overall sensitivity while reducing interobserver variability in adults (75).

\section{Magnetic Resonance Imaging (MRI)}

MRI is the preferred test for imaging the SB at diagnosis because it can detect changes that are characteristic of IBD and estimate both the extent of intestinal inflammation and the degree of 
damage (stricturing or penetrating disease). It is not extremely sensitive for subtle luminal disease that does not involve thickening of the bowel wall or significant increased intensity. Distension of the small bowel loops can be obtained by administering solution with nonabsorbable substances such as polyethylene glycol or sorbitol solution given by mouth (MRE), or via nasoenteric intubation (MR enteroclysis). MR enteroclysis is minimally superior to MRE yet is more invasive and therefore should not be used in children (76). Several variables of mucosal inflammation have been proposed, most notably bowel wall thickening, intensity enhancement of the bowel, engorgement of mesenteric vessels (ie, Comb sign), enlarged lymph nodes, and fatty infiltration of the mesentery (77). The presence of wall thickening and decreased luminal diameter may indicate stenotic disease, especially when prestenotic dilatation is visible. The detection of a stricture with a thickened hypointense bowel wall and no significant contrast-enhancement indicates a long-standing fibrotic stricture, which would not benefit from medical treatment $(78,79)$. Sinus tracts and fistulae appear as fluid-containing tracts with associated peripheral enhancement. MR also can depict enteroenteric fistulae that often form a complex network between closely adherent SB loops. An alternative protocol using only $150 \mathrm{~mL}$ of total fluid $(50 \mathrm{~mL}$ of lactulose in $100 \mathrm{~mL}$ of water) is extremely attractive, and compared prospectively with BS follow-through (SBFT) and endoscopy/histology (80). A systematic review of 11 relevant studies with 496 cases of suspected PIBD has confirmed that MRE is sensitive and specific for diagnosis of PIBD and that it should supersede conventional fluoroscopy as the SB imaging technique in centers with appropriate expertise (81). Metaanalysis of the 6 comparable studies gave a pooled sensitivity and specificity for MRE detection of active terminal ileal CD of $84 \%$ and $97 \%$, respectively $(81)$.

Pelvic MRI is recommended for the evaluation of patients with CD with suspected or proven perianal involvement. It allows definition of the extent and location of perianal fistulas and abscesses, thus providing critical information for both surgical management and for assessment of response to medical therapy (82).

\section{WCE (Video)}

WCE is the best alternative to MRE for investigating the SB, and it detects mucosal abnormalities. The main advantages of WCE are the ability to visualize the entire SB with minimal discomfort (83) and to detect mucosal lesions with a higher sensitivity than MRE. The main limitations are the inability to detect complications, the risk of capsule retention, an inability to control capsule movement, a high rate of incidental findings (ie, lower specificity), and the need to assess patency of the SB before the test. Contraindications include intestinal strictures, previous abdominal surgery (relative), severe disease with systemic features, and children $<1$ year $(84,85)$. For children unable to swallow the capsule, a specifically designed device enables introduction of the capsule during upper endoscopy into the duodenum (86).

In a meta-analysis in PIBD, the diagnostic yield for WCE ranged from $58 \%$ to $72 \%$, whereas it was $0 \%$ to $33 \%$ for SBFT and $0 \%$ to $61 \%$ for ileocolonoscopy (87). In a prospective pediatric controlled study in 20 children with suspected SB CD with either normal $(n=15)$ or nonspecific findings $(n=5)$ on conventional imaging, WCE use confirmed the diagnosis of CD in 12 (60\%) (88). A prospective, blinded 4-way comparison trial of WCE, CT, ileocolonoscopy, and SBFT in adults revealed a diagnostic sensitivity of $83 \%, 82 \%, 74 \%$, and $65 \%$, respectively, whereas the specificity of WCE (53\%) was significantly lower than that of all other tests (100\%) (89).

\section{BAE}

BAE including double-balloon enteroscopy (DBE) and single-balloon enteroscopy (SBE) has evolved over recent years and has progressively replaced push and surgically assisted enteroscopy (90). The role of SBE and DBE in the initial diagnostic workup of children with suspected $C D$ is extremely limited. The advantage of BAE over WCE for diagnosis includes visualization of lesions and facilitation of biopsy taking. Successful DBE has been reported to be safe and effective in a review of 5 pediatric series (91) whereas the experience with SBE is even more limited (92). Recently, 2 studies on the use of SBE in pediatric patients with suspected or established CD were performed. In 16 patients with suspected $\mathrm{CD}$ and unspecific findings at traditional endoscopy, and where WCE was diagnostic of CD only in 3, SBE with histology allowed a definite CD diagnosis in 12 (93); this usefulness was confirmed in a further series of 20 unclear pediatric cases (94). Spiral enteroscopy is an innovative technique for performing deep enteroscopy but no data reporting use in children are presently available.

\section{Special Circumstances}

\section{Recommendation}

An evaluation for primary immune deficiency should be performed in all cases of infantile IBD (diagnosed $<2$ years of age). [EL3b RGC]

\section{Practice Points}

1. Physicians evaluating patients with infantile IBD or recurrent infections should maintain a high index of suspicion for other causes of inflammation in the bowel.

2. An evaluation for allergic colitis should be considered in infantile IBD.

3. Children with symptoms of possible IBD developing after solid organ transplantation should be investigated for "de novo IBD."

The differential diagnoses of a child presenting with signs and symptoms of IBD is extensive, and it is beyond the scope of this article to delineate all possible conditions and infections that may mimic IBD. Several noninfectious disorders may also present with an IBD-like disease.

\section{Allergic Disorders}

Allergic colitis may mimic UC particularly in infants, but also in children beyond infancy (95). Eosinophilic gastroenteritis after infancy may mimic $\mathrm{CD}$ with ulcerations, skip lesions reaching from the stomach to the colon and, unlike its infantile counterpart, is uncommonly associated with allergy (96). Negative tests for specific immunoglobulin $\mathrm{E}$ against food allergens do not exclude allergic colitis or eosinophilic disorders (97). Under the appropriate circumstances, a cow's-milk protein elimination diet and-if symptoms resolve or improve - a challenge procedure may be justified in infants before drug treatment for IBD is started (98). IBD can also present initially as an eosinophilic predominant disease at diagnosis (99).

\section{PIDs}

GI manifestations such as colitis or Crohn-like disease are well recognized in patients with PID affecting the innate or adaptive immune system. There may be a diagnostic dilemma if the GI symptoms are the first or only manifestation of PID. Patients may be diagnosed and treated as CD or UC before the diagnosis of a PID has 
TABLE 4. Alarm signs and symptoms for primary immunodeficiency

Positive family history of primary immunodeficiency

Consanguineous parents or $>2$ family members with early-onset IBD

Infantile $(<2$ years) IBD

Severe, therapy-refractory IBD, particularly with perianal/rectovaginal disease/abscesses

Recurrent infections in the absence of immunosuppressant drugs (particularly pulmonary disease and skin abscesses)

Neutropenia, thrombocytopenia, or abnormal immune status (Ig levels) in the absence of immunosuppressant drugs

Nail dystrophy and hair abnormalities (trichorrhexis nodosa)

Skin abnormalities (congenital eczema, albino)

$\mathrm{IBD}=$ inflammatory bowel disease; $\mathrm{Ig}=$ immunoglobulin.

been entertained or demonstrated $(100,101)$. In these patients, IBD treatment options may be inappropriate or even harmful. Monogenetic immune disorders involving the interleukin-10 axis or XIAP gene presenting with intestinal or perianal disease can be proven or disproven by genetic or functional testing (101-103). A high degree of suspicion for PID is required in infantile-onset IBD or if the history shows any of the alarm symptoms or signs listed in Table 4 because PIDs may manifest as IBD during childhood. It is beyond the scope of this article to provide guidelines for the diagnostic workup of PID.

IBD can develop during immunosuppressive treatment, as is the case after solid organ transplantation (this is sometimes referred to as de novo IBD). Cases of IBD-like colitis have been described posttransplantation in children and in adults $(104,105)$. The incidence of IBD after transplantation is at least 10 times higher than the incidence of IBD in the general population and has been associated with the use of tacrolimus and Epstein-Barr virus infection. The workup for posttransplant IBD and its management are similar to those for classical IBD; however, every effort should be made to exclude opportunistic infections in this setting (104).

\section{PART 3: DIAGNOSTIC ALGORITHM FOR SUSPECTED PEDIATRIC IBD}

An algorithm based on the above recommendations, with grounding in the practice points and text, is presented as Figure 1, and achieved $>80 \%$ consensus within the expert group.

\section{CONCLUSIONS}

These revised Porto criteria for the diagnosis of PIBD have been developed to meet present challenges and developments in PIBD, and have placed evidence within the context of experience of European PIBD experts. Although the concept of the diagnostic workup has not changed, the revised Porto criteria are based on a robust methodological approach and the incorporation of the Paris phenotypic classification of PIBD (8); delineation of atypical phenotypes of PIBD; consideration of the advances in diagnostic imaging modalities, capsule endoscopy, and serological and fecal biomarkers; and a novel evidence-based approach to the definition of IBD-U. The document has been endorsed by the ESPGHAN.

\section{QUALIFYING STATEMENT}

These criteria may be revised as necessary to account for the changes in technology, new data, or other aspects of clinical practice. They are intended to be an educational tool to assist clinicians in providing care to patients, but they are not a rule and should not be construed as establishing a legal standard of care or as encouraging, advocating, requiring, or discouraging any particular treatment. Clinical decisions in any particular case involve a complex analysis of the patient's condition and available courses of action. Therefore, clinical considerations may require taking a course of action that varies from these criteria.

\section{REFERENCES}

1. IBD Working Group of the European Society for Paediatric Gastroenterology Hepatology and Nutrition. Inflammatory bowel disease in children and adolescents: recommendations for diagnosis- the Porto criteria. J Pediatr Gastroenterol Nutr 2005;41:1-7.

2. Van Limbergen J, Russell RK, Drummond HE, et al. Definition of phenotypic characteristics of childhood-onset inflammatory bowel disease. Gastroenterology 2008;135:1114-22.

3. Vernier-Massouille G, Balde M, Salleron J, et al. Natural history of pediatric Crohn's disease: a population-based cohort study. Gastroenterology 2008; 135:1106-13.

4. Gower-Rousseau C, Dauchet L, Vernier-Massouille G, et al. The natural history of pediatric ulcerative colitis: a population-based cohort study. Am J Gastroenterol 2009;104:2080-8.

5. Levine A, de Bie CL, Turner D, et al. Atypical disease phenotypes in paediatric ulcerative colitis: 5-year analyses of the EUROKIDS registry. Inflamm Bowel Dis 2013;19:370-7.

6. Bousvaros A, Antonioli DA, Colletti RB, et al. Differentiating ulcerative colitis from Crohn disease in children and young adults: report of a working group of the North American Society for Pediatric Gastroenterology, Hepatology, and Nutrition and the Crohn's and Colitis Foundation of America. J Pediatr Gastroenterol Nutr 2007;44:65374.

7. Turner D, Levine A, Escher JC, et al. Joint ECCO and ESPGHAN evidence-based consensus guidelines on the management of pediatric ulcerative colitis. J Pediatr Gastroenterol Nutr 2012;55:340-61.

8. Levine A, Griffiths A, Markowitz J, et al. Pediatric modification of the Montreal classification of inflammatory bowel disease: the Paris classification. Inflamm Bowel Dis 2011;17:1314-21.

9. Silverberg MS, Satsangi J, Ahmad T, et al. Toward an integrated clinical, molecular and serological classification of inflammatory bowel disease: report of a Working Party of the 2005 Montreal World Congress of Gastroenterology. Can J Gastroenterol 2005;19 (suppl A):5-36.

10. Gupta N, Bostrom AG, Kirschner BS, et al. Presentation and disease course in early- compared to later-onset pediatric Crohn's disease. Am J Gastroenterol 2008;103:2092-8.

11. Guariso G, Gasparetto M, Visona Dalla Pozza L, et al. Inflammatory bowel disease developing in paediatric and adult age. $J$ Pediatr Gastroenterol Nutr 2010;51:698-707.

12. Timmer A, Behrens R, Buderus S, et al. Childhood onset inflammatory bowel disease: predictors of delayed diagnosis from the CEDATA German-language pediatric inflammatory bowel disease registry. J Pediatr 2011;158:467-473.e2.

13. Kugathasan S, Judd RH, Hoffmann RG, et al. Epidemiologic and clinical characteristics of children with newly diagnosed inflammatory bowel disease in Wisconsin: a statewide population-based study. J Pediatr 2003;143:525-31.

14. Sawczenko A, Sandhu BK. Presenting features of inflammatory bowel disease in Great Britain and Ireland. Arch Dis Child 2003;88:9951000.

15. Jose FA, Garnett EA, Vittinghoff E, et al. Development of extraintestinal manifestations in pediatric patients with inflammatory bowel disease. Inflamm Bowel Dis 2009;15:63-8.

16. Stange EF, Travis SP, Vermeire S, et al. European evidence-based consensus on the diagnosis and management of ulcerative colitis: definitions and diagnosis. J Crohn's Colitis 2008;2:1-23.

17. Geboes K, Van Eyken P. Inflammatory bowel disease unclassified and indeterminate colitis: the role of the pathologist. J Clin Pathol 2009;62:201-5.

18. Glickman JN, Bousvaros A, Farraye FA, et al. Pediatric patients with untreated ulcerative colitis may present initially with unusual morphologic findings. Am J Surg Pathol 2004;28:190-7. 
19. Rajwal SR, Puntis JW, McClean P, et al. Endoscopic rectal sparing in children with untreated ulcerative colitis. J Pediatr Gastroenterol Nutr 2004:38:66-9.

20. Markowitz J, Kahn E, Grancher K, et al. Atypical rectosigmoid histology in children with newly diagnosed ulcerative colitis. Am J Gastroenterol 1993;88:2034-7.

21. Kim B, Barnett JL, Kleer CG, et al. Endoscopic and histological patchiness in treated ulcerative colitis. Am J Gastroenterol 1999; 94:3258-62.

22. Bernstein CN, Shanahan F, Anton PA, et al. Patchiness of mucosal inflammation in treated ulcerative colitis: a prospective study. Gastrointest Endosc 1995;42:232-7.

23. Washington K, Greenson JK, Montgomery E, et al. Histopathology of ulcerative colitis in initial rectal biopsy in children. Am J Surg Pathol 2002;26:1441-9.

24. Robert ME, Tang L, Hao LM, et al. Patterns of inflammation in mucosal biopsies of ulcerative colitis: perceived differences in pediatric populations are limited to children younger than 10 years. Am J Surg Pathol 2004;28:183-9.

25. Tobin JM, Sinha B, Ramani P, et al. Upper gastrointestinal mucosal disease in pediatric Crohn disease and ulcerative colitis: a blinded, controlled study. J Pediatr Gastroenterol Nutr 2001;32:443-8.

26. Xin W, Greenson JK. The clinical significance of focally enhanced gastritis. Am J Surg Pathol 2004;28:1347-51.

27. Goldstein N, Dulai M. Contemporary morphologic definition of backwash ileitis in ulcerative colitis and features that distinguish it from Crohn disease. Am J Clin Pathol 2006;126:365-76.

28. Haskell H, Andrews CW Jr, Reddy SI, et al. Pathologic features and clinical significance of "backwash" ileitis in ulcerative colitis. Am J Surg Pathol 2005;29:1472-81.

29. Perisic VN, Filipovic D. Ileoscopy and its clinical role in the assessment of backwash ileitis in children with ulcerative pancolitis: Belgrade experience. J Pediatr Gastroenterol Nutr 1988;7:146-8.

30. Newman SL. Ileoscopy, colonoscopy, and backwash ileitis in children with inflammatory bowel disease: quid pro quo? J Pediatr Gastroenterol Nutr 1987;6:325-7.

31. Lennard-Jones JE. Classification of inflammatory bowel disease. Scand J Gastroenterol 1989;24 (suppl 170):2-6.

32. Rowland M, Fleming P, Bourke B. Looking in the mouth for Crohn's disease. Inflamm Bowel Dis 2010;16:332-7.

33. Campbell H, Escudier M, Patel P, et al. Distinguishing orofacial granulomatosis from Crohn's disease: two separate disease entities? Inflamm Bowel Dis 2011;17:2109-15.

34. Keljo DJ, Markowitz J, Langton C, et al. Course and treatment of perianal disease in children newly diagnosed with Crohn's disease. Inflamm Bowel Dis 2009;15:383-7.

35. Escher JC, Stoof TJ, van Deventer SJH, et al. Successful treatment of metastatic Crohn disease with infliximab. J Pediatr Gastroenterol Nutr 2002;34:420-3.

36. de Bie CL, Paerregaard A, Kolacek S, et al. Disease phenotype at diagnosis in pediatric Crohn's disease: 5 year analyses of the EUROKIDS registry. Inflamm Bowel Dis 2013;19:378-85.

37. Rubio CA, Orrego A, Nesi G, et al. Frequency of epithelioid granulomas in colonoscopic biopsy specimens from paediatric and adult patients with Crohn's colitis. J Clin Pathol 2007;60:1268-72.

38. Matsui T, Yao T, Sakurai T, et al. Clinical features and pattern of indeterminate colitis: Crohn's disease with ulcerative colitis-like clinical presentation. J Gastroenterol 2003;38:647-55.

39. Alexander F, Sarigol S, DiFiore J, et al. Fate of the pouch in 151 pediatric patients after ileal pouch anal anastomosis. J Pediatr Surg 2003;38:78-82.

40. Melmed GY, Elashoff R, Chen GC, et al. Predicting a change in diagnosis from ulcerative colitis to Crohn's disease: a nested, casecontrol study. Clin Gastroenterol Hepatol 2007;5:602-8.

41. Langholz E, Munkholm P, Krasilnikoff PA, et al. Inflammatory bowel diseases with onset in childhood. Clinical features, morbidity, and mortality in a regional cohort. Scand J Gastroenterol 1997;32:13947.

42. Yantiss RK, Farraye FA, O'Brien MJ, et al. Prognostic significance of superficial fissuring ulceration in patients with severe "indeterminate" colitis. Am J Surg Pathol 2006;30:165-70.
43. Li DJ, Freeman A, Miles KA, et al. Can 99Tcm HMPAO leucocyte scintigraphy distinguish between Crohn's disease and ulcerative colitis? Br J Radiol 1994;67:472-7.

44. Xin W, Brown PI, Greenson JK. The clinical significance of focal active colitis in pediatric patients. Am J Surg Pathol 2003; 27:1134-8.

45. Ruemmele FM, Targan SR, Levy G, et al. Diagnostic accuracy of serological assays in pediatric inflammatory bowel disease. Gastroenterology 1998;115:822-9.

46. Hoffenberg EJ, Fidanza S, Sauaia A. Serologic testing for inflammatory bowel disease. J Pediatr 1999;134:447-52.

47. Markowitz J, Kugathasan S, Dubinsky M, et al. Age of diagnosis influences serologic responses in children with Crohn's disease: a possible clue to etiology? Inflamm Bowel Dis 2009;15:714-9.

48. Dubinsky MC, Lin YC, Dutridge D, et al. Serum immune responses predict rapid disease progression among children with Crohn's disease: immune responses predict disease progression. Am J Gastroenterol 2006;101:360-7.

49. Russell RK, Ip B, Aldhous MC, et al. Anti-Saccharomyces cerevisiae antibodies status is associated with oral involvement and disease severity in Crohn disease. J Pediatr Gastroenterol Nutr 2009;48: $161-7$.

50. Bartůnková J, Kolárová I, Sedivá A, et al. Antineutrophil cytoplasmic antibodies, anti-Saccharomyces cerevisiae antibodies, and specific IgE to food allergens in children with inflammatory bowel diseases. Clin Immunol 2002;102:162-8.

51. Joossens S, Reinisch W, Vermeire S, et al. The value of serologic markers in indeterminate colitis: a prospective follow-up study. Gastroenterology 2002;122:1242-7.

52. Iltanen S, Tervo L, Halttunen T, et al. Elevated serum anti-I2 and antiOmpW antibody levels in children with IBD. Inflamm Bowel Dis 2006;12:389-94.

53. Davis MK, Andres JM, Jolley CD, et al. Antibodies to Escherichia coli outer membrane porin $\mathrm{C}$ in the absence of anti-Saccharomyces cerevisiae antibodies and anti-neutrophil cytoplasmic antibodies are an unreliable marker of Crohn disease and ulcerative colitis. $J$ Pediatr Gastroenterol Nutr 2007;45:409-13.

54. Ashorn S, Honkanen T, Kolho KL, et al. Fecal calprotectin levels and serological responses to microbial antigens among children and adolescents with inflammatory bowel disease. Inflamm Bowel Dis 2009; 15:199-205.

55. Mack DR, Langton C, Markowitz J, et al. Laboratory values for children with newly diagnosed inflammatory bowel disease. Pediatrics 2007;119:1113-9.

56. Sidler MA, Leach ST, Day AS. Fecal S100A12 and fecal calprotectin as noninvasive markers for inflammatory bowel disease in children. Inflamm Bowel Dis 2008;14:359-66.

57. Sipponen T, Savilahti E, Kolho KL, et al. Crohn's disease activity assessed by fecal calprotectin and lactoferrin: correlation with Crohn's disease activity index and endoscopic findings. Inflamm Bowel Dis 2008; 14:40-6.

58. Shaoul R, Sladek M, Turner D, et al. Limitations of fecal calprotectin at diagnosis in untreated pediatric Crohn's disease. Inflamm Bowel Dis 2012;18:1493-7.

59. Khan K, Schwarzenberg SJ, Sharp H, et al. Role of serology and routine laboratory tests in childhood inflammatory bowel disease. Inflamm Bowel Dis 2002;8:325-9.

60. Cabrera-Abreu JC, Davies P, Matek Z, et al. Performance of blood tests in diagnosis of inflammatory bowel disease in a specialist clinic. Arch Dis Child 2004;89:69-71.

61. Turner D, Mack DR, Hyams J, et al. C-reactive protein (CRP), erythrocyte sedimentation rate (ESR) or both? A systematic evaluation in pediatric ulcerative colitis. J Crohns Colitis 2011;5:423-9.

62. Quail MA, Russell RK, Van Limbergen JE, et al. Faecal calprotectin complements routine laboratory investigations in diagnosing childhood inflammatory bowel disease. Inflamm Bowel Dis 2009; 15:756-9.

63. Henderson P, Casey A, Lawrence SJ, et al. The diagnostic accuracy of fecal calprotectin during the investigation of suspected pediatric inflammatory bowel disease. Am J Gastroenterol 2012; 107:941-9. 
64. Kapel N, Campeotto F, Kalach N, et al. Faecal calprotectin in term and preterm neonates. J Pediatr Gastroenterol Nutr 2010;51:542-7.

65. Henderson P, Anderson NH, Wilson DC. The diagnostic accuracy of fecal calprotectin during the investigation of suspected paediatric inflammatory bowel disease: a systematic review and meta-analysis. Am J Gastroenterol 2013doi:10.1038/ajg.2013.131[Epub ahead of print].

66. Trivedi PJ, Chapman RW. PSC, AIH and overlap syndrome in inflammatory bowel disease. Clin Res Hepatol Gastroenterol 2012; $36: 420-36$.

67. Husby S, Koletzko S, Korponay-Szabo IR, et al. European Society for Pediatric Gastroenterology, Hepatology, and Nutrition guidelines for the diagnosis of coeliac disease. J Pediatr Gastroenterol Nutr 2012; 54:136-60.

68. de Bie CI, Buderus S, Sandhu BK, et al. Diagnostic workup of paediatric patients with inflammatory bowel disease in Europe: results of a 5-year audit of the EUROKIDS registry. $J$ Pediatr Gastroenterol Nutr 2012;54:374-80.

69. van Assche G, Dignass A, Panes J, et al. The second European evidence-based Consensus on the diagnosis and management of Crohn's disease: definitions and diagnosis. J Crohns Colitis 2010; 4:7-27.

70. Paerregaard A. What does the IBD patient hide in the upper gastrointestinal tract? Inflamm Bowel Dis 2009;15:1101-4.

71. Lemberg DA, Clarkson CM, Bohane TD, et al. Role of esophagogastroduodenoscopy in the initial assessment of children with inflammatory bowel disease. J Gastroenterol Hepatol 2005;20: 1696-700.

72. Haber HP, Busch A, Ziebach R, et al. Bowel wall thickness measured by ultrasound as a marker of Crohn's disease activity in children. Lancet 2000;355:1239-40.

73. Maconi G, Greco S, Duca P, et al. Prevalence and clinical significance of sonographic evidence of mesenteric fat alterations in Crohn's disease. Inflamm Bowel Dis 2008;14:1555-61.

74. Bremner AR, Griffiths M, Argent JD, et al. Sonographic evaluation of inflammatory bowel disease: a prospective, blinded, comparative study. Pediatr Radiol 2006;36:947-53.

75. Migaleddu V, Quaia E, Scanu, et al. Inflammatory activity in Crohn's disease: CE-US. Abdom Imaging 2011;36:142-8.

76. Schreyer AG, Geissler A, Albrich H, et al. Abdominal MRI after enteroclysis or with oral contrast in patients with suspected or proven Crohn's disease. Clin Gastroenterol Hepatol 2004;2:491-7.

77. Chalian M, Ozturk A, Oliva-Hemker M, et al. MR enterography findings of inflammatory bowel disease in pediatric patients. Am J Roentgenol 2011;196:W810-6.

78. Durno CA, Sherman P, Williams T, et al. Magnetic resonance imaging to distinguish the type and severity of pediatric inflammatory bowel diseases. J Pediatr Gastroenterol Nutr 2000;30:170-4.

79. Siddiki HA, Fidler JL, Fletcher JG, et al. Prospective comparison of state-of-the-art MR enterography and CT enterography in small-bowel Crohn's disease. Am J Roentgenol 2009;193:113-21.

80. Giles E, Hanci O, McLean A, et al. Optimal assessment of paediatric IBD - a comparison study of MRI and barium follow through. J Paediatr Gastroenterol Nutr 2012;54:758-62.

81. Giles E, Barclay AR, Chippington S, et al. Systematic review: MRI for assessment of small bowel disease in paediatric Crohn's disease. Aliment Pharm Ther 2013;37:1121-31.

82. Essary B, Kim J, Anupindi S, et al. Pelvic MRI in children with Crohn disease and suspected perianal involvement. Pediatr Radiol 2007; 37:201-8.

83. Liao Z, Gao R, Xu C, et al. Indications and detection, completion, and retention rates of small-bowel capsule endoscopy: a systematic review. Gastrointest Endosc 2010;71:280-6.

84. Cheifetz AS, Kornbluth AA, Legnani P, et al. The risk of retention of the capsule endoscope in patients with known or suspected Crohn's disease. Am J Gastroenterol 2006;101:2218-22.
85. Fritscher-Ravens A, Scherbakov P, Bufler P, et al. The feasibility of wireless capsule endoscopy in detecting small intestinal pathology in children under the age of 8 years: a multicentre European study. Gut 2009;58:1467-72.

86. Cohen SA, Gralnek IM, Ephrath H, et al. The use of a patency capsule in pediatric Crohn's disease: a prospective evaluation. Dig Dis Sci 2011;56:860-5.

87. Cohen SA, Klevens AI. Use of capsule endoscopy in diagnosis and management of pediatric patients, based on meta-analysis. Clin Gastroenterol Hepatol 2011;9:490-6.

88. Guilhon de Araujo Sant'Anna AM, Dubois J, Miron MC, et al. Wireless capsule endoscopy for obscure small-bowel disorders: final results of the first pediatric controlled trial. Clin Gastroenterol Hepatol 2005;3:264-70.

89. Solem CA, Loftus EV Jr, Fletcher JG, et al. Small-bowel imaging in Crohn's disease: a prospective, blinded, 4-way comparison trial. Gastrointest Endosc 2008;68:255-66.

90. Pasha SF, Leighton JA, Das A, et al. Double-balloon enteroscopy and capsule endoscopy have comparable diagnostic yield in small-bowel disease: a meta-analysis. Clin Gastroenterol Hepatol 2008;6:671-6.

91. Lin TK, Erdman SH. Double-balloon enteroscopy: pediatric experience. J Pediatr Gastroenterol Nutr 2010;51:429-32.

92. Barth BA, Channabasappa N. Single-balloon enteroscopy in children: initial experience at a pediatric center. J Pediatr Gastroenterol Nutr 2010;51:680-4.

93. Di Nardo G, Oliva S, Aloi M, et al. Usefulness of single-balloon enteroscopy in pediatric Crohn's disease. Gastrointest Endosc 2012;75:80-6.

94. de Ridder L, Mensink PB, Lequin MH, et al. Single-balloon enteroscopy, magnetic resonance enterography, and abdominal US useful for evaluation of small-bowel disease in children with (suspected) Crohn's disease. Gastrointest Endosc 2012;75:87-94.

95. Ravelli A, Villanacci V, Monfredini C, et al. How patchy is patchy villous atrophy?: distribution pattern of histological lesions in the duodenum of children with celiac disease. Am J Gastroenterol 2010;105:2103-10.

96. Rothenberg ME. Eosinophilic gastrointestinal disorders (EGID). J Allergy Clin Immunol 2004;113:11-28.

97. Boyce JA, Assa'ad A, Burks AW, et al. Guidelines for the diagnosis and management of food allergy in the United States: summary of the NIAID-sponsored expert panel report. J Am Diet Assoc 2011;111:1727.

98. Koletzko S, Niggemann B, Arato A, et al. Diagnostic approach and management of cow's-milk protein allergy in infants and children: ESPGHAN GI Committee Practical Guidelines. J Pediatr Gastroenterol Nutr 2012;55:221-9.

99. Katsanos KH, Zinovieva E, Lambri E, et al. Eosinophilic-Crohn overlap colitis and review of the literature. J Crohns Colitis 2011;5:256-61.

100. Freudenberg F, Wintergerst U, Roesen-Wolff A, et al. Therapeutic strategy in p47-phox deficient chronic granulomatous disease presenting as inflammatory bowel disease. J Allergy Clin Immunol 2010; 125:943-6.

101. Horn PC, Belohradsky BH, Urban C, et al. Two new families with Xlinked inhibitor of apoptosis deficiency and a review of all 26 published cases. J Allergy Clin Immunol 2011;127:544-6.

102. Glocker EO, Kotlarz D, Boztug K, et al. Inflammatory bowel disease and mutations affecting the interleukin-10 receptor. $N$ Engl J Med 2009;361:2033-45.

103. Kotlarz D, Beier R, Murugan D, et al. Loss of interleukin-10 signaling and infantile inflammatory bowel disease: implications for diagnosis and therapy. Gastroenterol 2012;143:347-55.

104. Verdonk RC, Dijkstra G, Haagsma EB, et al. Inflammatory bowel disease after liver transplantation: risk factors for recurrence and de novo disease. Am J Transplant 2006;6:1422-9.

105. Dehghani SM, Eshraghian A, Bahador A, et al. De novo inflammatory bowel disease after pediatric orthotopic liver transplant: a case report. Exp Clin Transplant 2009;7:145-8. 